\title{
Effect of Human Flavin-Containing Monooxygenase 3 Polymorphism on the Metabolism of Aurora Kinase Inhibitors
}

\section{Gianluca Catucci, Andrea Occhipinti, Massimo Maffei, Gianfranco Gilardi and Sheila J. Sadeghi *}

Department of Life Sciences and Systems Biology, University of Torino, Via Accademia Albertina 13, Torino 10123, Italy; E-Mails: gianluca.catucci@unito.it (G.C.); andrea.occhipinti@unito.it (A.O.); massimo.maffei@unito.it (M.M.); gianfranco.gilardi@unito.it (G.G.)

* Author to whom correspondence should be addressed; E-Mail: sheila.sadeghi@unito.it; Tel.: +39-011-670-4528; Fax: +39-011-670-4643.

Received: 9 October 2012; in revised form: 22 December 2012 / Accepted: 18 January 2013 / Published: 28 January 2013

Abstract: Aurora kinases were recently identified as a potential target in anticancer therapy and, amongst their available inhibitors, Tozasertib (VX-680) and Danusertib (PHA-739358) have been indicated as possible substrates of human flavin-containing monooxygenase 3 (hFMO3). Here we report the in vitro rate of oxidation of these drugs by wild-type hFMO3 and its polymorphic variant V257M. The conversion of Tozasertib and Danusertib to their corresponding metabolites, identified by LC-MS, by the purified wild-type and V257M hFMO3 show significant differences. In the case of Tozasertib, the V257M variant shows a catalytic efficiency, expressed as $k_{\text {cat }} / K_{\mathrm{m}}$, similar to the wild-type: $0.39 \pm 0.06 \mathrm{~min}^{-1} \mu \mathrm{M}^{-1}$ for $\mathrm{V} 257 \mathrm{M}$ compared to $0.33 \pm 0.04 \mathrm{~min}^{-1} \mu \mathrm{M}^{-1}$ for the wild type. On the other hand, in the case of Danusertib, V257M shows a $3.4 \times$ decrease in catalytic efficiency with $k_{\text {cat }} / K_{\mathrm{m}}$ values of $0.05 \pm 0.01 \mathrm{~min}^{-1} \mu \mathrm{M}^{-1}$ for $\mathrm{V} 257 \mathrm{M}$ and $0.17 \pm 0.03 \mathrm{~min}^{-1} \mu \mathrm{M}^{-1}$ for the wild type. These data reveal how a simple V257M substitution ascribed to a single nucleotide polymorphism affects the $N$-oxidation of relevant anticancer drugs, with important outcome in their therapeutic effects. These findings demonstrate that codon 257 is important for activity of the hFMO3 gene and the codon change $\mathrm{V}$ to $\mathrm{M}$ has an effect on the catalytic efficiency of this enzyme.

Keywords: phase I; drug metabolism; FMO3; polymorphism; enzyme kinetics; VX-680; PHA-739358; LC-MS 


\section{Introduction}

Human flavin-containing monooxygenase 3 (hFMO3) is the second most important Phase I drug metabolising enzyme after cytochromes P450. It is expressed in the adult human liver where it catalyses the NADPH-dependent $N$ - and $S$-oxidation of drugs and xenobiotics [1]. Previously it has been reported that there is a 10- to 20-fold inter-individual difference in FMO expression that may contribute to drug response [2-4]. Furthermore, the existence of polymorphisms affecting hFMO3-dependent metabolism has also been documented [5], with several studies showing reduced activity with some common variants.

Several polymorphic variants of hFMO3 have been identified among which V257M (rs2266782), E158K (rs2266780), and E308G (rs1736557) are the most commonly distributed. Inter-individual and inter-ethnic variability in hFMO3 expression and enzyme activity is primarily due to genetics, therefore, making it possible to correlate hFMO3 allelic variants to drug-induced side effects or drug response. It has been previously shown that minor allele frequencies of E158K, V257M, and E308G vary greatly among different ethnic populations with distinct genetic backgrounds [6]. Moreover, allelic variation within the hFMO3 gene can also influence a person's drug responsiveness [7] with important differences in the therapeutic response of life-saving drugs such as anticancer drugs.

Aurora A, Aurora B, and Aurora C are serine-threonine kinases that regulate mitotic events. The first two are the best studied and were assigned different roles in mitosis [8]: Aurora A was found to be over-expressed in many human tumors like breast, colorectal, and ovarian cancer [8], and Aurora B is also over-expressed in various types of cancer [9,10]. Specific inhibitors of Aurora kinases, such as VX-680, MLN8054, AZD1152, R766, R763, and PHA-739358, have been designed and screened for their inhibitory activity in cancer events [11].

The VX-680 inhibitor, also known as Tozasertib, was originally discovered by Harrington and co-workers [12], and it is an inhibitor of Aurora-A, Aurora-B, and Aurora-C kinases with inhibition constants $\left(\mathrm{Ki}_{\text {app }}\right)$ of $0.6,18$ and $4.6 \mathrm{nM}$, respectively $[12,13]$.

Rational design of the pyrrolopyrazole identified from the combinatorial expansion of the 1,4,5,6-tetrahydropyrrolo[3,4-c]pyrazoles bound to the pocket of the target led to the discovery of the PHA-739358 inhibitor, also called Danusertib [14,15]. This compound was also found to inhibit several tyrosine kinases such as Abl, Ret, Trk-A, and FGFR-1 that are involved in many types of cancer such as leukemia, thyroid, prostate, and breast carcinoma [14,15].

Tozasertib is metabolised into the $N$-desmethyl product by CYP3A4 and CYP2C8, and into the $\mathrm{N}$-oxide product by CYP3A4 and hFMO3, as previously demonstrated by Ballard and co-workers [16]. Their study highlighted the combined contribution of hFMO3 and P450 3A4 to the formation of the $\mathrm{N}$-oxide metabolite in human liver microsomes.

Knowledge of genetic polymorphism and its possible related functional changes is increasingly considered critical for both drug discovery and drug development [17,18]. Since Tozasertib and Danusertib have been indicated as substrates of the drug metabolizing flavin-containing monooxygenases, in this work we measure the kinetic parameters of the wild type hFMO3 (WT) and V257M polymorphic variant with these Aurora kinase inhibitors. 


\section{Results and Discussion}

In order to determine the kinetic parameters related to the metabolism of Tozasertib and Danusertib by hFMO3, both WT and the V257M polymorphic variant were heterologously expressed in Echerichia coli and purified as previously described [19-22]. Both proteins showed a single band on SDS-PAGE gel with a yield of around $12 \mathrm{mg}$ of protein per litre of culture. A molar ratio of more than 91\% holo protein was determined spectrophotometrically [21] for both proteins. Positive control reactions, with benzydamine and methimazole as substrates, were performed to confirm the catalytic activities of the enzyme preparations.

We have chosen the V257M and not the other two common SNPs (single nucleotide polymorphism) due to the fact that residue 257 is located in the "insert" region of the hFMO3 gene which is absent in the bacteria and yeast FMO amino acid sequences [23]. As a consequence, this "insert" region is not present in the two published crystal structures of FMO and, therefore, the exact location of this amino acid in relation to the active site of this enzyme can only be hinted at by ab initio modeling. We have previously generated a model of hFMO3 [19], however in our model the location of residue 257 is not very close to the FAD catalytic center, similar to the modeling results of Rettie and colleagues [23].

In a previous study we had shown the turnover of the WT hFOM3 in the presence of both Danusertib and Tozasertib using HPLC analysis [21]. In the current report, the metabolites of the enzymatic reaction of both WT and V257M polymorphic variant with Danusertib and Tozasertib were identified by LC-MS. In each case, the occurrence of the enzymatic oxidative reaction was confirmed by the molecular shift of 16 atomic mass units (amu) of both substrates. The oxidation sites were confirmed through comparison with LC-MS fragmentation profiles of substrates and observed metabolites.

\subsection{Tozasertib Metabolism by hFMO3}

In 2007, Ballard and co-workers [16] identified the metabolites of Tozasertib generated in human hepatocytes that were mainly the $\mathrm{N}$-oxide and the desmethyl products. They went on to characterize the hepatic enzymes responsible and found that hFMO3 appreciably (50\%-60\%) contributed to the $\mathrm{N}$-oxidation of Tozasertib in human liver microsomes together with cytochrome P450 3A4. Following in their footsteps, we investigated the metabolism of Tozasertib using the purified WT hFMO3, and identified the metabolite by LC-MS. The suggested fragmentation profile of Tozasertib (Figure 1A) includes the molecular ion $[\mathrm{M}+\mathrm{H}]^{+} 465.2$ and subsequent 397.2 and 340.0 in $\mathrm{MS}_{(3)}$ as cleavage of $\mathrm{C}_{4} \mathrm{O}$ group and pyridine ring, respectively. On the other hand, Tozasertib- $N$-oxide fragmentation (Figure 1B) shows the molecular ion $[\mathrm{M}+\mathrm{H}]^{+} 481.2$, the cleavage of $\mathrm{N}$-oxide pyridine ring (394.1) first and the cleavage of $\mathrm{C}_{4} \mathrm{O}$ in the last fragmentation step.

The same enzymatic reactions followed by LC-MS analyses were carried out for the V257M polymorphic variant in order to test whether the variant was not only capable of metabolising Tozasertib, but also resulted in the same $N$-oxide metabolite. 
Figure 1. (a) Fragmentation profile of Tozasertib showing the molecular ion $[\mathrm{M}+\mathrm{H}]^{+}$ 465.2 and subsequent 397.2 and 340.0 in $\mathrm{MS}_{(3)}$ as cleavage of $\mathrm{C}_{4} \mathrm{O}$ group and pyridine ring, respectively; (b) Tozasertib- $N$-oxide fragmentation showing the molecular ion $[\mathrm{M}+\mathrm{H}]^{+} 481.2$, the cleavage of $N$-oxide pyridine ring (394.1) first and the cleavage of $\mathrm{C}_{4} \mathrm{O}$ in the last fragmentation step.

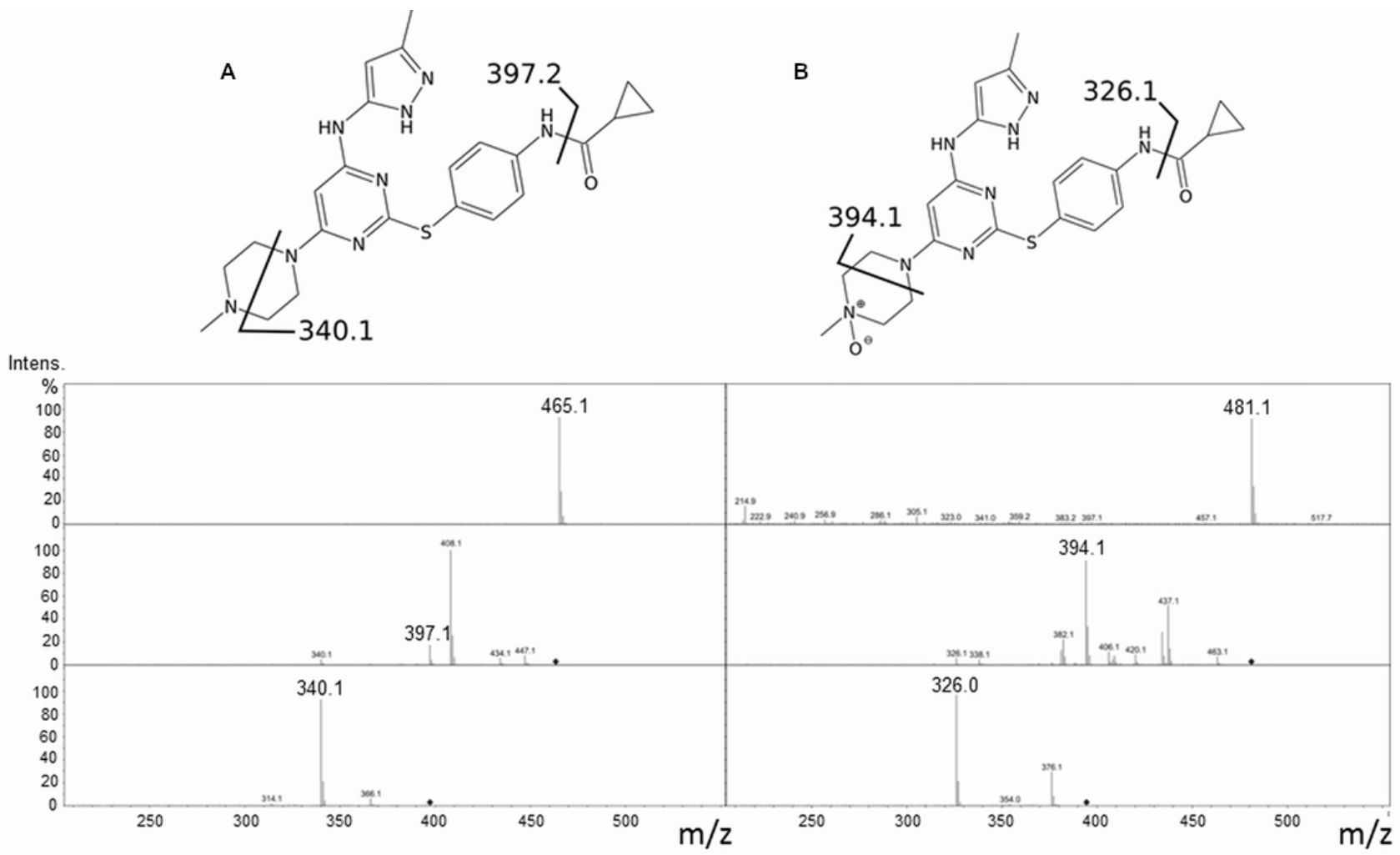

\subsection{Danusertib Metabolism by hFMO3}

The major route of Danusertib metabolism involves the formation of $N$-oxide product by the action of hFMO3 forming an inactive metabolite. A possible influence of a SNP in the hFMO3 gene, on the metabolism of Danusertib, was proposed by Steeghs and co-workers in 2010 [24] when they showed a different clearance of the drug from the body in a patient heterozygous for V257M. Since the latter correlation between Danusertib pharmacokinetics and genetic variation was based on the data from a single patient, we thought the role of V257M in the metabolism of Danusertib warranted further investigation and decided to check the kinetics of Danusertib metabolism with the purified hFMO3 V257M polymorphic variant. To this end, initially in vitro experiments were set up for the identification of the $\mathrm{N}$-oxide product by LC-MS and subsequently followed by the determination of the kinetic parameters of the enzymatic reactions.

For the LC-MS experiments, purified WT and V257M hFMO3 were incubated with NADPH and Danusertib and the enzymatic reaction terminated after $10 \mathrm{~min}$, and the resulting metabolites identified as described in the experimental section. Mass spectrometry analysis for Danusertib and its $N$-oxide showed, at the first fragmentation step, the loss of the common methoxy group $\left[\mathrm{M}-\mathrm{CH}_{3} \mathrm{OH}\right]^{+}$ (443.2 and 459.2 for Danusertib and its $N$-oxide). Their $\mathrm{MS}_{(2)}$ fragmentation generates ion 203.0 for Danusertib and the 16 amu-shifted fragment at 219.0 for the $N$-oxide product (Figure 2A,B). 
Moreover, the latter showed a fragmentation ion from $\mathrm{N}$-oxide pyridine ring at 160.0 that limits the $\mathrm{N}$-oxidation site to the quaternary amine on the pyridine ring.

Figure 2. (a) Fragmentation profile of Danusertib showing the molecular ion $[\mathrm{M}+\mathrm{H}]^{+}$ 475.2 and subsequent 443.2 as loss of the common methoxy group $\left[\mathrm{M}-\mathrm{CH}_{3} \mathrm{OH}\right]^{+}$at the first fragmentation step; (b) Fragmentation profile of Danusertib $\mathrm{N}$-oxide showing the molecular ion $[\mathrm{M}+\mathrm{H}]^{+} 459.2$ as loss of the common methoxy group $\left[\mathrm{M}-\mathrm{CH}_{3} \mathrm{OH}\right]^{+}$at the first fragmentation step.

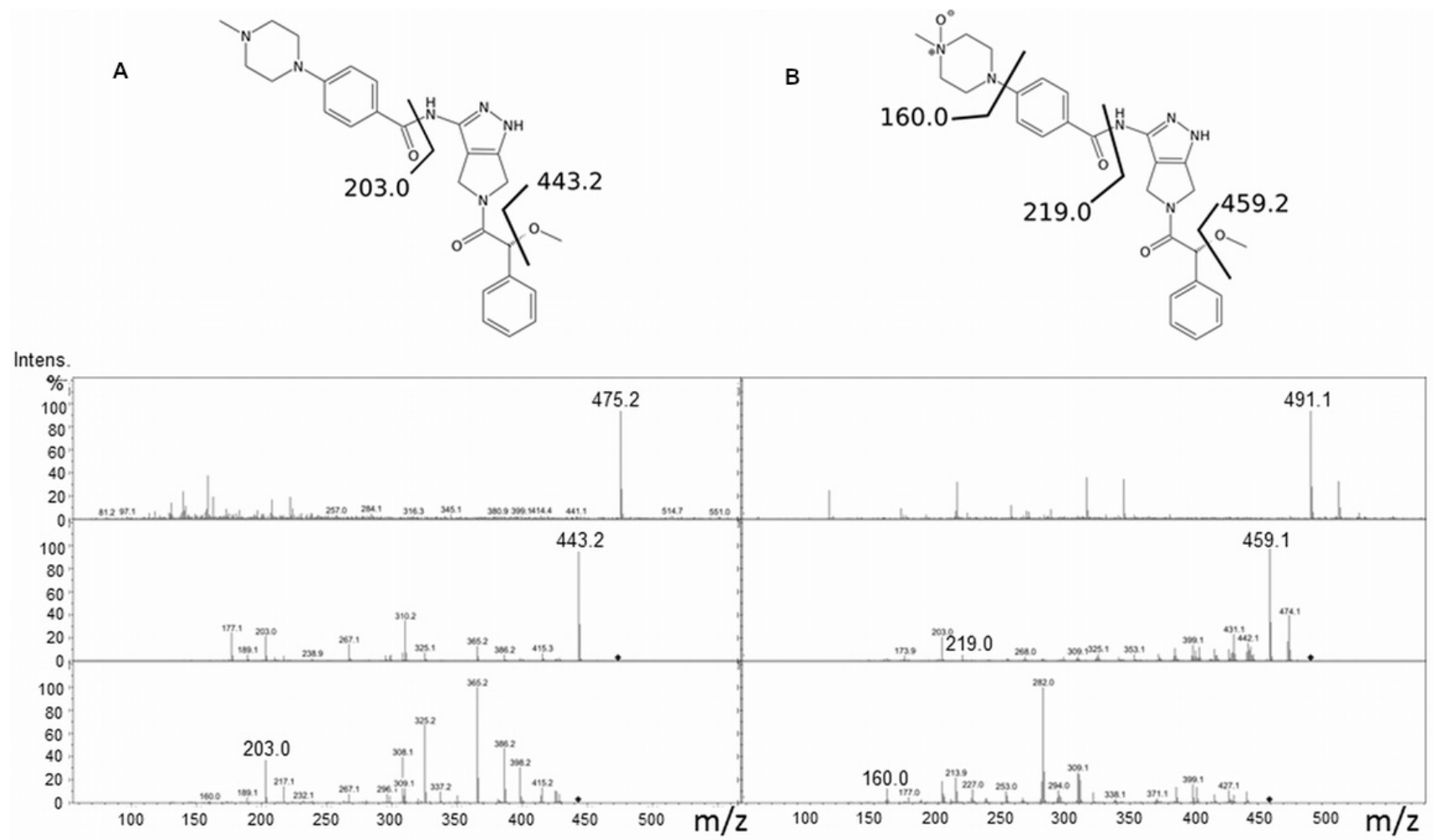

\subsection{Kinetic Measurements of hFMO3 with Aurora Kinase Inhibitors}

Enzymatic reactions were carried out with the purified WT and V257M polymorphic variant in the presence of NADPH and increasing amounts of each substrate as reported in the experimental section. Kinetic parameters for $N$-oxygenation of Tozasertib were determined by nonlinear regression analysis and showed a $K_{\mathrm{m}}$ of $23.8 \mu \mathrm{M}$, a $k_{\text {cat }}$ of $9.3 \mathrm{~min}^{-1}$ in the case of the wild-type enzyme and a $K_{\mathrm{m}}$ value of $12.9 \mu \mathrm{M}$, a $k_{\text {cat }}$ of $4.3 \mathrm{~min}^{-1}$ for the polymorphic variant $\mathrm{V} 257 \mathrm{M}$. The $k_{\text {cat }}$ and $K_{\mathrm{m}}$ values for V257M were approximately half of that of the wild-type (Figure $3 \mathrm{~A}, \mathrm{~B}$ ) and the $k_{\text {cat }}$ for the wild-type enzyme was at least twice as much of the $k_{\text {cat }}$ for V257M. When the ratios of $k_{\text {cat }} / K_{\mathrm{m}}$ are compared, both enzymes seem to be equally efficient in transforming Tozasertib into the $N$-oxide product. The $K_{\mathrm{m}}$ values measured are slightly higher when compared to the data published by Ballard and co-workers [16], probably because they specifically address the substrate binding to hFMO3 and do not consider the role of cytochrome P450 3A4 that also contributes to the overall $N$-oxide metabolite of Tozasertib. Moreover, for Tozasertib the $\mathrm{V}_{\max }$ of wild type hFMO3 was $536 \mathrm{pmol} / \mathrm{min} / \mathrm{mg}$ FMO3 while V257M hFMO3 $\mathrm{V}_{\max }$ resulted in $248 \mathrm{pmol} / \mathrm{min} / \mathrm{mg}$ FMO3 (Table 1) in good agreement with the data published by Ballard and co-workers [16]. 
Figure 3. (a) Michaelis-Menten kinetics for the formation of the $N$-oxide metabolite of Tozasertib and Danusertib with wild type (A and C, respectively), and V257M (B and D, respectively) hFMO3. Reactions were carried out with $0.17 \mu \mathrm{M}$ of each purified enzyme in $50 \mathrm{mM}$ potassium phosphate buffer $\mathrm{pH} 7.4$ and $0.5 \mathrm{mM}$ NADPH in the presence of increasing amounts of Tozasertib $(0-300 \mu \mathrm{M})$ and Danusertib $(0-200 \mu \mathrm{M})$, at $37{ }^{\circ} \mathrm{C}$ for $10-15 \mathrm{~min}$.

A

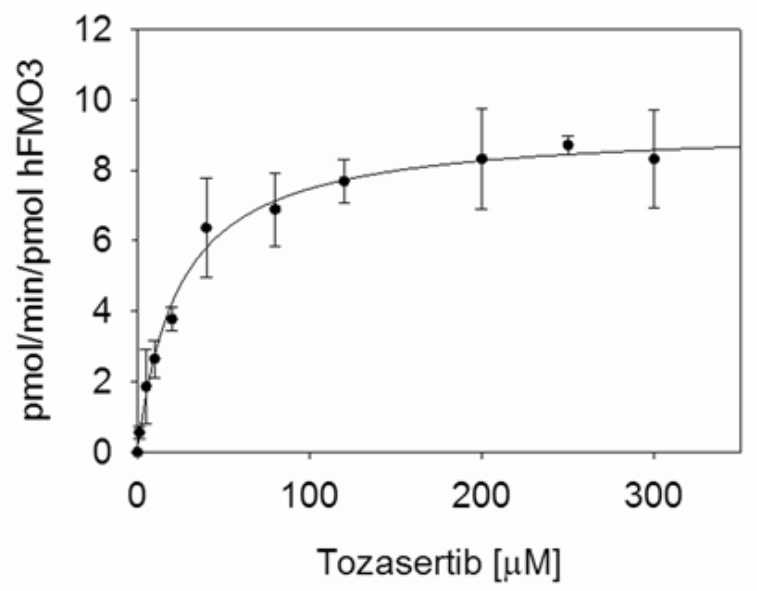

C

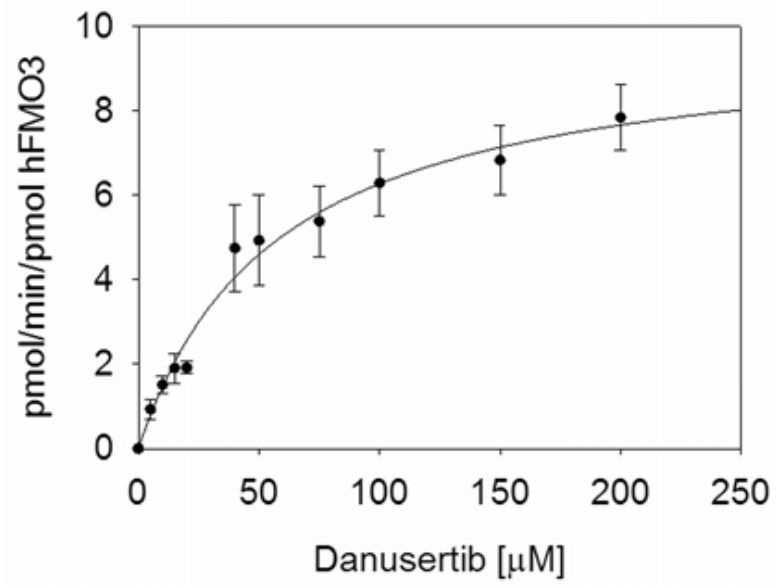

B

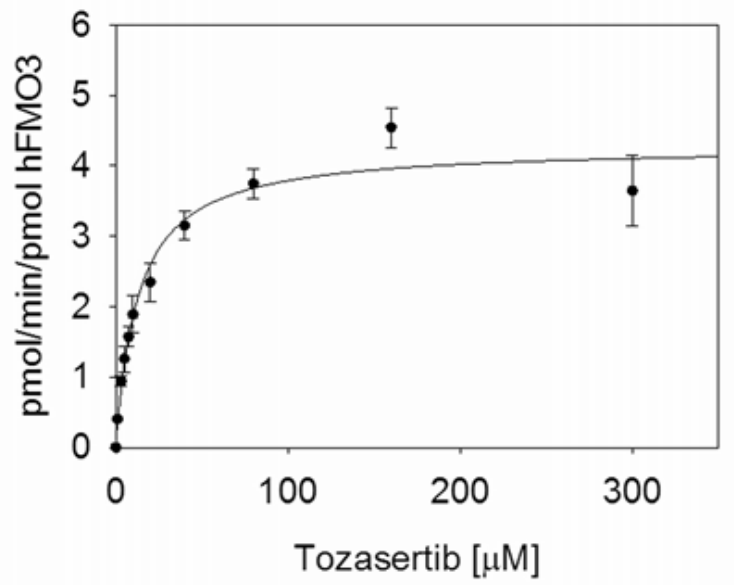

D

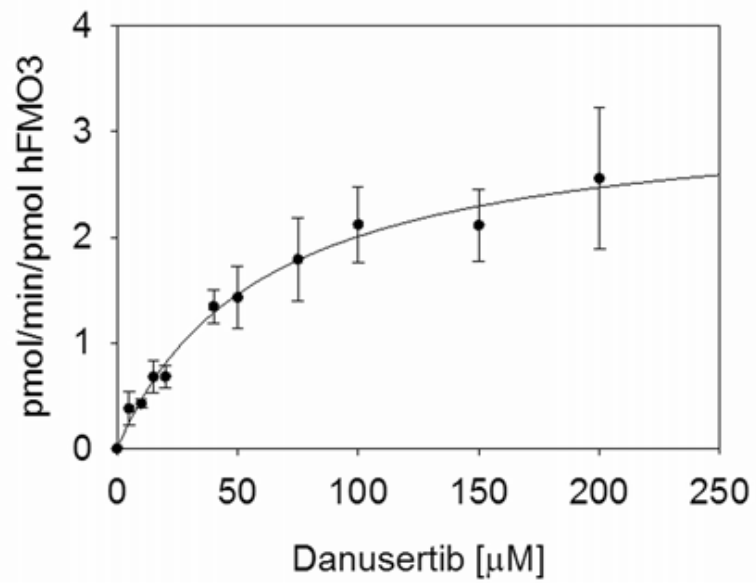

Kinetic parameters for $N$-oxygenation of Danusertib were also determined by nonlinear regression analysis, and showed a $K_{\mathrm{m}}$ of $57.3 \mu \mathrm{M}$, a $\mathrm{V}_{\max }$ of $0.57 \mathrm{nmol} \mathrm{min}{ }^{-1} \mathrm{mg} \mathrm{FMO3}$, and $k_{\text {cat }}$ of $9.9 \mathrm{~min}^{-1}$ for the wild-type enzyme and a $K_{\mathrm{m}}$ of $60.1 \mu \mathrm{M}$, a $\mathrm{V}_{\max }$ of $0.18 \mathrm{nmol} \mathrm{min}{ }^{-1} \mathrm{mg}$ FMO3 and $k_{\text {cat }}$ of $3.2 \mathrm{~min}^{-1}$ for V257M (Figure 3C,D). The $K_{\mathrm{m}}$ value for the polymorphic variant V257M was very similar to that of the wild-type indicating that Danusertib binding is similar in the two proteins. More interestingly the $\mathrm{V}_{\max }$ in the case of $\mathrm{V} 257 \mathrm{M}$ was approximately three times less than the wild-type (Table 1), showing a decreased turnover rate that yields a significantly lower turnover number. The $k_{\text {cat }} / K_{\mathrm{m}}$ ratio is three times higher for the wild type hFMO3, which means that the variant V257M is less efficient in metabolizing Danusertib. 
Table 1. Kinetic analysis of Tozasertib and Danusertib metabolism by WT and V257M polymorphic variant of hFMO3.

\begin{tabular}{cccccccccc}
\hline \multicolumn{9}{c}{ WT hFMO3 } & \multicolumn{3}{c}{ V257M hFMO3 } \\
\hline & $K_{\mathrm{m}}$ & $\mathrm{V}_{\max }$ & $k_{\text {cat }}$ & $k_{\text {cal }} / K_{\mathrm{m}}$ & $K_{\mathrm{m}}$ & $\mathrm{V}_{\text {max }}$ & $k_{\text {cat }}$ & $k_{\text {cat }} / K_{\mathrm{m}}$ \\
& $\mu \mathrm{M}$ & $\mathrm{nmol} / \mathrm{min} / \mathrm{mg} \mathrm{FMO3}$ & $\min ^{-1}$ & $\min ^{-1} \mu \mathrm{M}^{-1}$ & $\mu \mathrm{M}$ & $\mathrm{nmol} / \mathrm{min} / \mathrm{mg} \mathrm{FMO3}$ & $\mathrm{nmol} / \mathrm{min} / \mathrm{mg} \mathrm{FMO3} \mathrm{min}^{-1} \mu M^{-1}$ \\
Tozasertib & $23.8 \pm 3.4$ & $0.54 \pm 0.02$ & $9.3 \pm 0.3$ & $0.39 \pm 0.06$ & $12.9 \pm 1.3$ & $0.25 \pm 0.01$ & $4.3 \pm 0.1$ & $0.33 \pm 0.04$ \\
Danusertib & $57.3 \pm 8.3$ & $0.57 \pm 0.03$ & $9.9 \pm 0.6$ & $0.17 \pm 0.03$ & $60.1 \pm 11.4$ & $0.18 \pm 0.01$ & $3.2 \pm 0.3$ & $0.05 \pm 0.01$ \\
\hline
\end{tabular}

\section{Experimental Section}

\subsection{Reagents}

FAD, acetonitrile, methanol, NADPH, Verapamil and salts were purchased from Sigma-Aldrich (Milan, Italy). VX-680 and PHA-739358 were purchased from Aurogene (Rome, Italy).

\subsection{Recombinant FMO3 Protein Preparation}

WT hFMO3 was cloned in the expression vector pJL2 using the two restriction enzymes, XbaI and HindIII, as previously reported [19,21]. QuikChange Site-Directed Mutagenesis Kit (Stratagene, Torino, Italy) was used to obtain the V257M polymorphic variant using the following two primers (mutation site in bold):

\section{5' CT GAC TGG TTG TAC ATG AAG CAG ATG AAT GC 3' 5' GC ATT CAT CTG CTT CAT GTA CAA CCA GTC AG 3'}

Subsequently the presence of the correct mutation was confirmed by sequencing the entire clone.

Wild type and V257M hFMO3 were heterologously expressed in E. coli JM109 cells and grown $24 \mathrm{~h}$ post-induction. Both proteins were purified from the membrane fractions using $\mathrm{Ni}$ affinity chromatography. Spectra of the fractions eluted with $40 \mathrm{mM}$ histidine were recorded using a diode array HP-8453E spectrophotometer. FAD-containing fractions with the characteristic absorption peaks at 375 and $442 \mathrm{~nm}$ were pooled and exchanged with storage buffer (100 mM potassium phosphate buffer $\mathrm{pH} 7.4,20 \%$ glycerol and $1 \mathrm{mM}$ EDTA) by $30 \mathrm{kDa}$ cutoff Amicon membranes (Millipore, Billercia, MA, USA) and stored at $-20^{\circ} \mathrm{C}$.

The concentration of hFMO3 was determined by spectroscopy with the peak absorbance at $450 \mathrm{~nm}$ and an extinction coefficient of $11900 \mathrm{M}^{-1} \mathrm{~cm}^{-1}$. This value was also used for determination of the enzyme concentration under non-denaturing conditions. The yield of the purified hFMO3 protein was determined using both absorbance at $280 \mathrm{~nm}$ (calculated extinction coefficient of $87520 \mathrm{M}^{-1} \mathrm{~cm}^{-1}$ ) and Bradford assay.

\subsection{Enzyme Assays}

$N$-oxygenation of Tozasertib and Danusertib was evaluated by incubating a mixture of $0.17 \mu \mathrm{M}$ of purified enzyme in $50 \mathrm{mM}$ potassium phosphate buffer ( $\mathrm{pH} 7.4$ ), $0.5 \mathrm{mM} \mathrm{NADPH}$, and increasing amounts of substrate in a final volume of $0.20 \mathrm{~mL}$. The enzyme and NADPH were mixed first and the reaction was initiated by the addition of substrate, preventing thermal degradation of the enzyme. Incubations were carried out at $37^{\circ} \mathrm{C}$ for $10 \mathrm{~min}$ in the case of Danusertib, and $15 \mathrm{~min}$ in the case of 
Tozasertib: the linearity of product formation was confirmed with purified hFMO3 preparations for $20 \mathrm{~min}$. The incubations were terminated by the addition of $0.10 \mathrm{~mL}$ of ice-cold methanol and $150 \mu \mathrm{L}$ verapamil as internal standard (IS).

\subsection{LC-MS Analyses of Danusertib, Tozasertib and Corresponding N-oxides}

Prior to LC-MS analysis the reaction mixtures were filtered through a Microcon centrifugal membrane with $3 \mathrm{kDa}$ cutoff (Millipore, Billercia, MA, USA). Substrates and their metabolites were separated using Agilent 1200 HPLC (Agilent Technologies Inc., Santa Clara, CA, USA) equipped with a narrow bore reversed-phase column Zorbax Eclipse XDB-C18, $2.1 \times 150 \mathrm{~mm}, 3.5 \mu \mathrm{m}$ (Agilent Technologies, Santa Clara, CA, USA) and using a binary solvent system composed of double distilled water with $0.01 \%$ formic acid (A), and acetonitrile (ACN) with $0.1 \%$ formic acid (B) at flow rate of $0.2 \mathrm{~mL} \mathrm{~min}^{-1}$. The initial mobile phase was composed of $20 \%$ of solvent B and it was increased to $70 \%$ over a period of $3 \mathrm{~min}$ and kept $4 \mathrm{~min}$ for isocratic separations of substrate, metabolite and internal standard (IS). Finally, the organic solvent was increased to $100 \%$ in $30 \mathrm{~s}$ for washing the column. Before follower injection, initial mobile phase was re-established for $10 \mathrm{~min}$.

Prior to MS detection, the column eluate was monitored on-line with UV detection at $250 \mathrm{~nm}$ wavelength for substrates and their metabolites, and $280 \mathrm{~nm}$ for IS. The MS analyses were made by a 6330 Series Ion Trap Mass Spectrometer (Bruker Daltonik GmbH, Bremen, Germany) equipped with an electrospray ionization source.

Analyses for fragmentation spectra were conducted by ESI-MS/MS operating in full scan from 50 to $900 \mathrm{~m} / \mathrm{z}$. Spectra were acquired in positive mode with ion spray voltage at $1.2 \mathrm{kV}$, nebulizer gas $\left(\mathrm{N}_{2}\right)$ at 15 psi and $5 \mathrm{~L} \mathrm{~min}^{-1}$, dry temperature at $325^{\circ} \mathrm{C}$, and $1.00 \mathrm{~V}$ fragmentation amplitude.

For quantitative purpose, standards and samples were analysed by LC-ESI-MS/MS in MRM mode using the above-indicated parameters. The monitored mass transitions were $\mathrm{m} / \mathrm{s} 465.2 \rightarrow 408.1$, $481.2 \rightarrow 437.1,475.2 \rightarrow 443.2,491.2 \rightarrow 459.2$ and $455.3 \rightarrow 303.1$ for Tozasertib, Tozasertib- $N$-oxide, Danusertib, Danusertib- $N$-oxide and Verapamil, respectively. Spectral data were processed using data analysis software of 6300 Series Ion Trap LC/MS 4.0 (Bruker Daltonik GmbH, Bremen, Germany). Metabolite formation was quantified by comparing peak area ratios (metabolite/internal standard) of four data sets of incubations for each Michaelis-Menten curve to ratios obtained from a standard curve containing known amounts of the internal standard verapamil.

\section{Conclusions}

In conclusion, Tozasertib is metabolised by hFMO3 to the expected $N$-oxide. The V257M polymorphic variant displays little difference in the $N$-oxidation of this drug, so since cytochrome P450 3A4 and hFMO3 appear to be important in the latter reaction [16] the inter-individual variations related to the metabolism of this drug can only be due to genetic or environmental factors influencing the activity of cytochrome P450 3A4. On the other hand, Danusertib is also metabolised by hFMO3 to the corresponding $N$-oxide, but its metabolism is markedly influenced by the V257M polymorphism showing a 3.4 times lower catalytic efficiency compared to the wild type enzyme implying that inter-individual variations of the hFMO3 gene can result in decreased activity towards this drug. 
Finally, the V257M substitution of hFMO3 represents a good example of how this SNP can influence the $\mathrm{N}$-oxidation of relevant anticancer drugs. These findings demonstrate the need for further research into the consequences of flavin-containing monooxygenase genetic polymorphism in drug metabolism.

\section{Acknowledgments}

The authors wish to thank Maria Razalan for her help in the purification of the variant protein.

\section{Conflict of Interest}

The authors declare no conflict of interest.

\section{References}

1. Krueger, S.K.; Williams, D.E. Mammalian FMO: Structure/function, genetic polymorphism and role in drug metabolism. Pharmacol. Ther. 2005, 106, 357-387.

2. Overby, L.H.; Carver, G.C.; Philpot, R.M. Quantitation and kinetic properties of hepatic microsomal and recombinant flavin-containing monooxygenase 3 and 5 from humans. Chem. Biol. Interact. 1997, 106, 29-45.

3. Yeung, C.K.; Lang, D.H.; Thummel, K.E.; Rettie, A.E. Immunoquantitation of FMO1 in human liver, kidney and intestine. Drug Metab. Dispos. 2000, 28, 1107-1111.

4. Koukouritaki, S.B.; Poch, M.T.; Henderson, M.C.; Siddens, L.K.; Krueger, S.K.; VanDyke, J.E.; Williams, D.E.; Pajewski, N.M.; Wang, T.; Hines, R.N. Identification and functional analysis of common human flavin-containing monooxygenase 3 genetic variants. J. Pharm. Exp. Therap. 2007, 320, 266-273.

5. Koukouritaki, S.B.; Hines, R.N. Flavin-containing monooxygenase genetic polymorphism: Impact on chemical metabolism and drug development. Pharmacogenomics 2005, 6, 807-822.

6. Cashman, J.R.; Zhang, J.; Leushner, J.; Braun, A. Population distribution of human flavin-containing monooxygenase form 3: Gene polymorphisms. Drug Metab. Dispos. 2001, 29, 1629-1637.

7. Cashman, J.R.; Akerman, B.R.; Forrest, S.M.; Treacy, E.P. Population-specific polymorphisms of the human FMO3 gene: Significance for detoxication. Drug Metab. Dispos. 2000, 2, 169-173.

8. Li, Y.; Zhang, Z.F.; Chen, J.; Huang, D.; Ding, Y.; Tan, M.H.; Qian, C.N.; Resau, J.H.; Kim, H.; Teh, B.T. VX-680/MK-0457, a potent and selective Aurora kinase inhibitor, targets both tumor and endothelial cells in clear cell renal cell carcinoma. Am. J. Transl. Res. 2000, 3, 296-308.

9. Gautschi, O.; Heighway, J.; Mack, P.C.; Purnell, P.R.; Lara, P.N., Jr.; Gandara, D.R. Aurora kinases as anticancer drug targets. Clin. Cancer Res. 2008, 14, 1639-1648.

10. Vader, G.; Lens, S.M. The Aurora kinase family in cell division and cancer. Biochim. Biophys. Acta 2008, 1786, 60-72.

11. Martinelli, G.; Iacobucci, I.; Papayannidis, C.; Soverini, S. New targets for $\mathrm{Ph}^{+}$leukaemia therapy. Best Pract. Res. Clin. Haematol. 2009, 3, 445-454. 
12. Harrington, E.A.; Bebbington, D.; Moore, J.; Rasmussen, R.K.; Ajose-Adeogun, A.O.; Nakayama, T.; Graham, J.A.; Demur, C.; Hercend, T.; Diu-Hercend, A.; et al. VX-680 A potent and selective small-molecule inhibitor of aurora kinases suppresses tumor growth in vivo. Nat. Med. 2004, 10, 262-267.

13. Kitzen, J.J.; de Jonge, M.J.; Verweij, J. Aurora kinase inhibitors. Crit. Rev. Oncol. Hemat. 2010, 2, 99-110.

14. Fancelli, D.; Moll, J.; Varasi, M.; Bravo, R.; Artico, R.; Berta, D.; Bindi, S.; Cameron, A.; Candiani, I.; Cappella, P.; et al. 1,4,5,6-Tetrahydropyrrolo[3,4-c]pyrazoles: Identification of a potent Aurora kinase inhibitor with a favorable antitumor kinase inhibition profile. J. Med. Chem. 2006, 49, 7247-7251.

15. Carpinelli, P.; Ceruti, R.; Giorgini, M.L.; Cappella, P.; Gianellini, L.; Croci, V.; Degrassi, A.; Texido, G.; Rocchetti, M.; Vianello, P.; et al. PHA-739358, a potent inhibitor of Aurora kinases with a selective target inhibition profile relevant to cancer. Mol. Cancer Ther. 2007, 12, 3158-3168.

16. Ballard, J.E.; Prueksaritanont, T.; Tang, C. Hepatic metabolism of MK-0457, a potent aurora kinase inhibitor: Interspecies comparison and role of human cytochrome P450 and flavin-containing monooxygenase. Drug Metab. Dispos. 2007, 9, 1447-1451.

17. Roses, A.D. Pharmacogenetics and drug development: The path to safer and more effective drugs. Nat. Rev. Genet. 2004, 5, 645-656.

18. Panicco, P.; Dodhia, V.R.; Fantuzzi, A.; Gilardi, G. First enzyme-based amperometric platform to determine the polymorphic response in drug metabolism by cytochromes P450. Anal. Chem. 2011, 83, 2179-2186.

19. Sadeghi, S.J.; Meirinhos, R.; Catucci, G.; Dodhia, V.R.; di Nardo, G.; Gilardi, G. Direct electrochemistry of drug metabolizing hFMO3: Electrochemical turnover of benzydamine and tamoxifen. J. Am. Chem. Soc. 2010, 132, 458-459.

20. Castrignanò, S.; Sadeghi, S.J.; Gilardi, G. Electro-catalysis by immobilised human flavin-containing monooxygenase isoform 3 (hFMO3). Anal. Bioanal. Chem. 2010, 398, 1403-1409.

21. Catucci, G.; Gilardi, G.; Jeuken, L.; Sadeghi, S.J. In vitro drug metabolism by $C$-terminally truncated human flavin-containing monooxygenase 3. Biochem. Pharmacol. 2012, 83, 551-558.

22. Castrignanò, S.; Sadeghi, S.J.; Gilardi, G. Entrapment of human flavin-containing monooxygenase 3 in the presence of gold nanoparticles: TEM, FTIR and electrocatalysis. Biochim. Biophys. Acta 2012, 1820, 2072-2078.

23. Yeung, C.K.; Adman, E.T.; Rettie, A.E. Functional characterization of genetic variants of human FMO3 associated with TMAU. Arch. Biochem. Biophys. 2007, 464, 251-259.

24. Steeghs, N.; Mathijssen, R.H.; Wessels, J.A.; de Graan, A.J.; van der Straaten, T.; Mariani, M.; Laffranchi, B.; Comis, S.; de Jonge, M.J.A.; Gelderblom, H.; et al. Influence of pharmacogenetic variability on the pharmacokinetics and toxicity of the aurora kinase inhibitor danusertib. Invest. New Drugs 2010, 5, 953-962.

(C) 2013 by the authors; licensee MDPI, Basel, Switzerland. This article is an open access article distributed under the terms and conditions of the Creative Commons Attribution license (http://creativecommons.org/licenses/by/3.0/). 